\title{
Dynamic Simulation and Analysis on Elevating Process of Elevating Equilibrator
}

\author{
Wanpeng Ma , Jisheng Ma and Lijun Cao \\ Artillery Engineering Department, Ordance Engineering College, Shijiazhuang, China
}

\begin{abstract}
The elevating equilibrator is used to control the elevating angel of the director of a rocket launcher. Based on an elevating equilibrator, a method for co-simulation is introduced with dynamic simulation software ADAMS and hydraulic simulation software EASY5. A three-dimensional model of the elevating equilibrator is developed with Solidworks and is imported into ADAMS. Furthermore, the hydraulic system model is established by EASY5. The interaction of mechanical system and hydraulic system is analyzed. The analysis indicates that the modeling method for co-simulation is effective and practical.
\end{abstract}

\section{Introduction}

The elevating equilibrator is a relatively complex action mechanism and used to control the elevating angel of the director of a rocket launcher. As the mechanism is simulated, the mechanical system and hydraulic system should be analyzed together. In this paper, the virtual prototype of the elevating equilibrator is established with ADAMS and hydraulic system model is established with EASY5 respectively. Then co-simulation is achieved by dynamic data exchange between two models [1]. Because of a large load generated when elevating equilibrator working, the dynamic simulation and analysis on elevating process of elevating equilibrator is of great significance [2].

\section{Mechanical and hydraulic system modeling}

\subsection{Elevating equilibrator modeling}

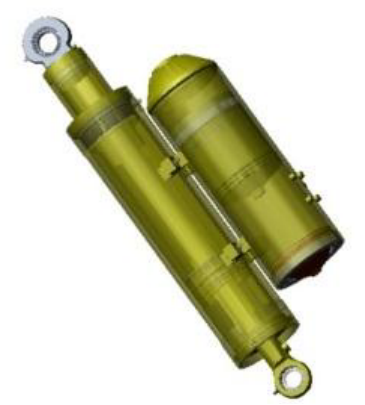

Figure 1. Elevating Equilibrator Model
The elevating equilibrator is constituted of hydraulic cylinders and energy accumulator. Significantly the hydraulic cylinder is composed of a fixed piston rod, a moving piston rod and sealing elements, and, the upward, downward and balance cavities are in the hydraulic cylinder. The accumulator includes a variable accumulator volume and an optional fluid tee-section that connects to adjacent components. The elevating equilibrator is shown in Fig. 1.

The elevating equilibrator model that is established and assembled in Solidworks software[3] is imported into ADAMS software and simplified on the basis of the dynamic relation. The dynamic simulation and analysis are in progress after the material properties defined and joints and load created. The virtual prototyping model is shown in Fig. 2.

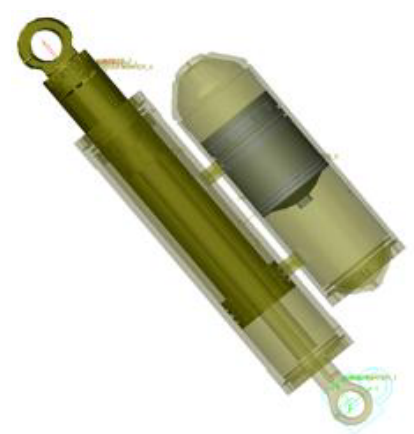

Figure 2. Simplified Model

\subsection{Hydraulic system modeling}

\subsubsection{Hydraulic system circuit}

Hydraulic oil flows into upward and downward cavity through the hydraulic system circuit, which controls the 
elevating process, and the location of machine is fixed by the hydraulic lock composed of two hydraulic control check valve. The circuit is established in EASY5 software, which includes the creating of hydraulic oil, pumps, sinks, cylinders and various control valves. Hydraulic system circuit is shown in Fig. 3.

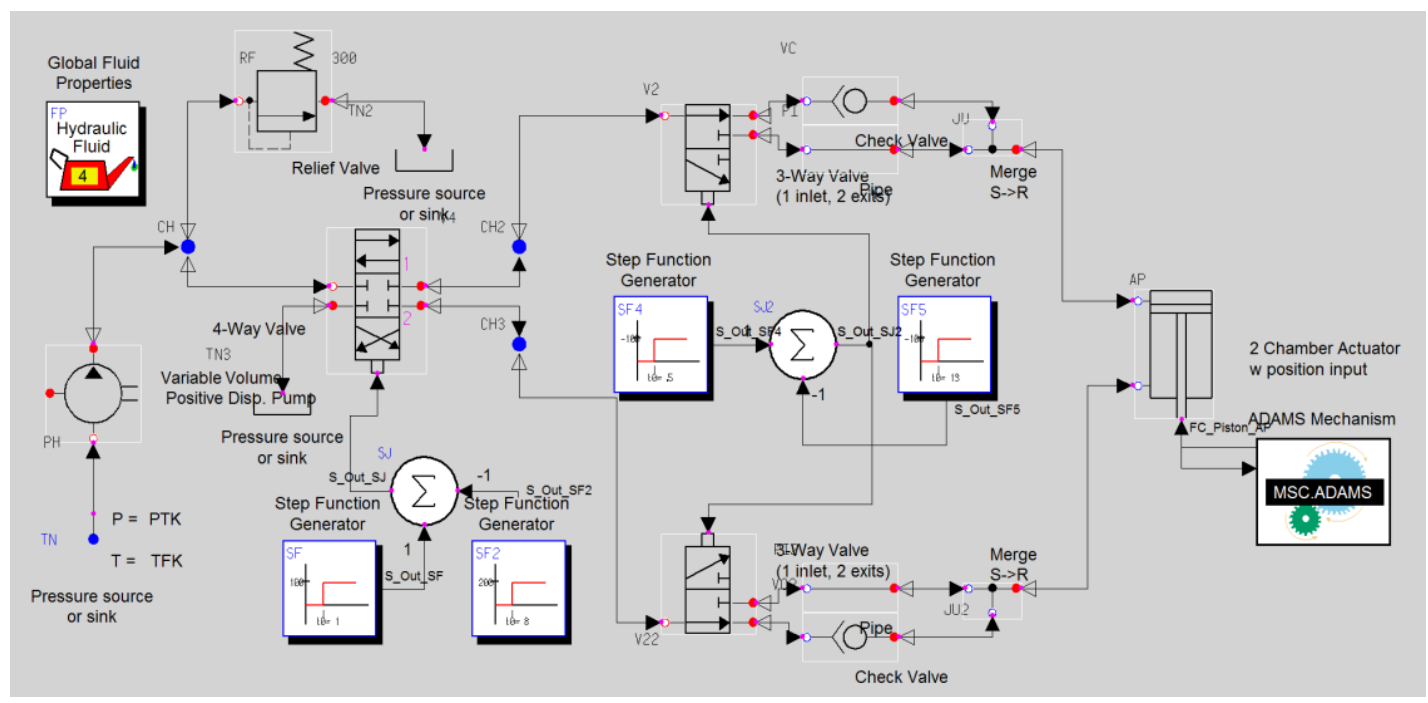

Figure 3. Hydraulic system modeling

In the hydraulic system model, the elevating process and the hydraulic lock are controlled by the 4-way valve and two 3-way valves respectively. The system pressure is limited for the relief valve, and energy is storage and reused by the energy accumulator.

\subsubsection{Parameter setting}

It is of significance to configure parameters of oil properties, power components, actuator components and various controllers. \#10 aircraft hydraulic oil (SH0358-1995) ${ }^{[4]}$ is chosen to be used in the circuit, and the fluid temperature(TC), ambient temperature and pressure are defined on basis of the working condition.

Force formula of the cylinder is:

$$
\mathrm{F}=P_{1} A_{1}-P_{2} A_{2}=P_{1} \frac{\pi D^{2}}{4}-P_{2} \frac{\pi\left(D^{2}-d^{2}\right)}{4}
$$

where $\mathrm{F}$ is the force of hydraulic cylinder, $P_{1} P_{2}$ stand for oil pressure of extend and retract chamber, and $A_{1} A_{2}$ stand for piston area of extend and retract chamber. The parameters of the cylinders and other components are designed, and the parameters are shown in Table 1.

Table 1. Parameters of Hydraulic Circuit

\begin{tabular}{|c|c|}
\hline PC_RF(bar) & 300 \\
\hline PFO_RF(bar) & 320 \\
\hline TC_RF(s) & 0.01 \\
\hline PCK_VC(bar) & 1 \\
\hline APE_AP(cm2) & 50 \\
\hline APR_AP(cm2) & 40 \\
\hline
\end{tabular}

\section{Co-simulation of ADAMS and EASY5}

New design variables are created in ADAMS software including an input variable (force) and two output variables (displacement, velocity) are shown in Table 2.

Table 2. State Variables Designed

\begin{tabular}{|c|c|c|}
\hline $\begin{array}{c}\text { State } \\
\text { Variable }\end{array}$ & Type & Meaning \\
\hline Force & Input & Force to Piston Rod \\
\hline Displacement & Output & Displacement of Piston rod \\
\hline velocity & Output & Velocity of Piston rod \\
\hline
\end{tabular}

And these variables are connected with ADAMS Mechanism Component (AD) in EASY5 software through the ADAMS/Control and EASY5/Extension interface. In Chosen Execution Mode, the Function Evaluation (no feed-through) is selected ${ }^{[5]}$

The ADAMS/View is started through the EASY5 Command Shell, and the control file of model (.cmd) and hydraulic control system file (.dll) are imported into virtual prototyping model. When interactive simulation was using, simulation process always failed because integral can not converge. Therefore the scripted simulation method is determined which controls integral steps by expression command ${ }^{[6]}$. Simulation integral step is 0.0005 before $13 \mathrm{~s}$ and 0.005 before $15 \mathrm{~s}$, which is determined by repeated simulation test.

\section{Results and analysis}

\subsection{Motion process analysis}

Starting from $1 \mathrm{~s}$, the piston rod rises at a constant speed and drops from $8 \mathrm{~s}$ at the same speed. When the 4-way valve is working, a shock will be engendered between hydraulic oil and both sides of piston rod. Because of that, at $1 \mathrm{~s}$ and $8 \mathrm{~s}$, the velocity of piston rod will change rapidly 
and stabilize in a very short period of time. At $13 \mathrm{~s}$, the hydraulic circuit is locked by the hydraulic lock, and the motion of piston rod is stopped. The simulation results of the velocity and displacement are shown in Fig.4 and Fig.5

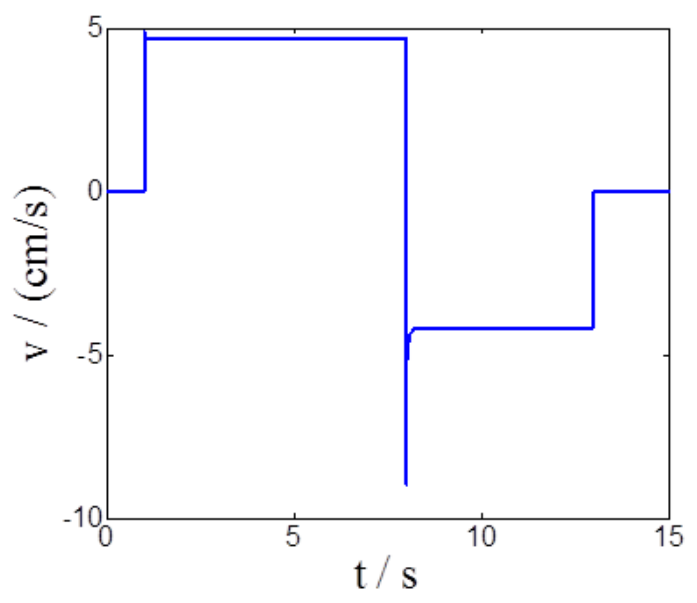

Figure 4. Velocity of Piston Rod

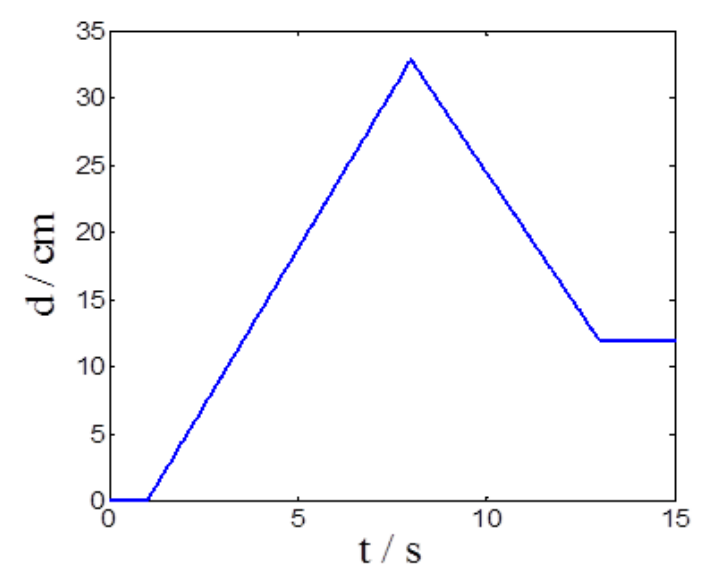

Figure 5. Displacement of Piston Rod

\subsection{Force analysis of piston rod}

In this simulation, the hydraulic oil pressure in the upward cavity and downward cavity are shown in Fig.6.

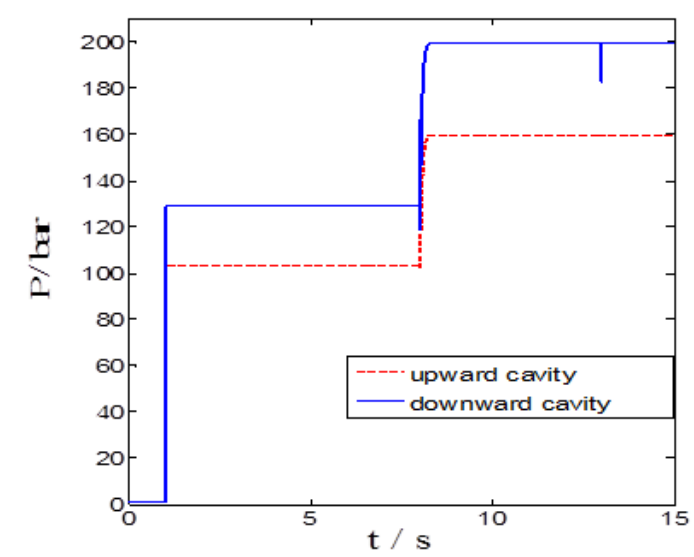

Figure 6. Changes of Pressure in Cylinder

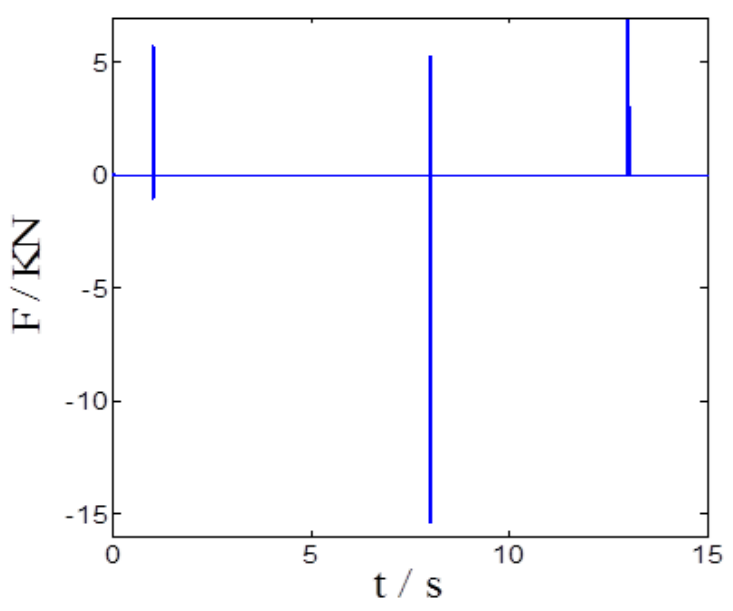

Figure 7. Shock Force of Oil Flow

When the direction of oil flow is changed by operate manual reversing valve, pressure of oil is changed rapidly at same time. At $13 \mathrm{~s}$, the hydraulic lock is closed up and pressure also is changed obviously. These changes in simulation results are in agreement with the actual working situation and these simulation values are credible and meaningful. The direction of oil flow changing, a shock between oil and piston rod is of significant impact for service life and dynamics performance of piston rod. The simulation results of shock force is shown in Fig.7, and at $1 \mathrm{~s}, 8 \mathrm{~s}$ and $13 \mathrm{~s}$ the shock force is produced due to the change of the oil flow direction and the closure of the hydraulic lock. Seen from Fig.7, the maximum shock force occurs at the moment of change in the direction of oil flow at $8 \mathrm{~s}$, and this condition is in agreement with the actual working situation. Based on the above simulation results and analysis, the virtual prototyping model and hydraulic control system model are effective and practical, and the simulation results are credible and available for reference.

\subsection{Flow analysis of relief valve and directional valve}

Flow is a very important parameter for the hydraulic control system. At the level of balance machine complicated system, the flow parameters are difficult to be measured. Through the above analysis, the preliminary confirmation of co-simulation model established is consistent with the actual working conditions. So the flow analysis for the complex components can be finished by co-simulation.

The flow parameters of the relief valve and the 4-way valve are shown in Fig.. 8, 9, 10. From the figure, the flow can be analysed, when the mechanism is stopped, the relief valve is fully open, the flow is to a maximum value of $49.09 \mathrm{~cm}^{\wedge} 3 / \mathrm{s}$. At the rising stage, the flow from port 1 of 4-way valve is positive, and the value of port 2 is negative. At the downward stage, the flow of port1 and port2 is just the opposite. 


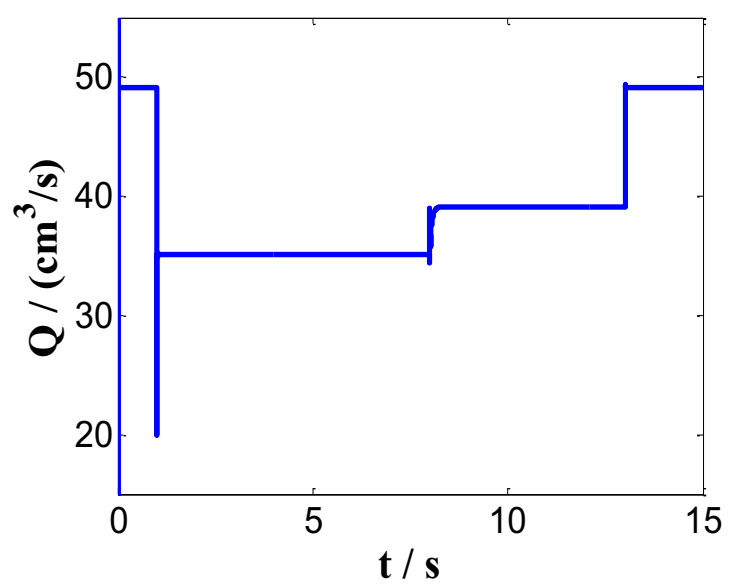

Figure 8. Flow of relief valve

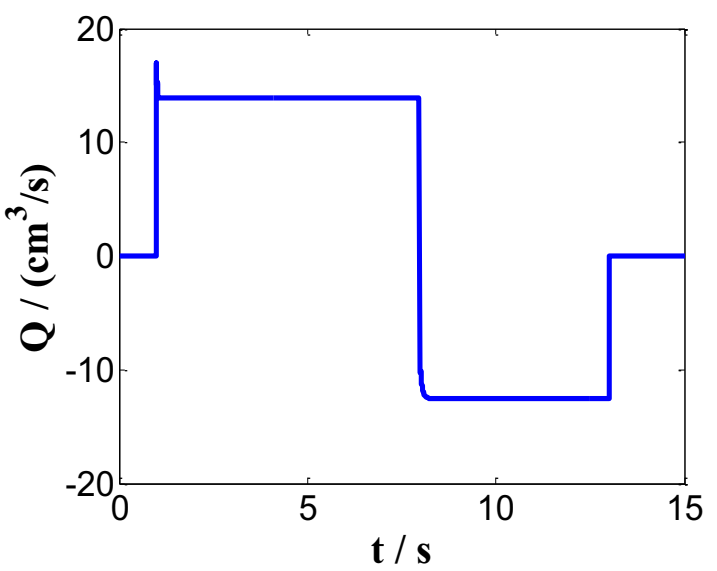

Figure 9. Flow of 4-way valve port1

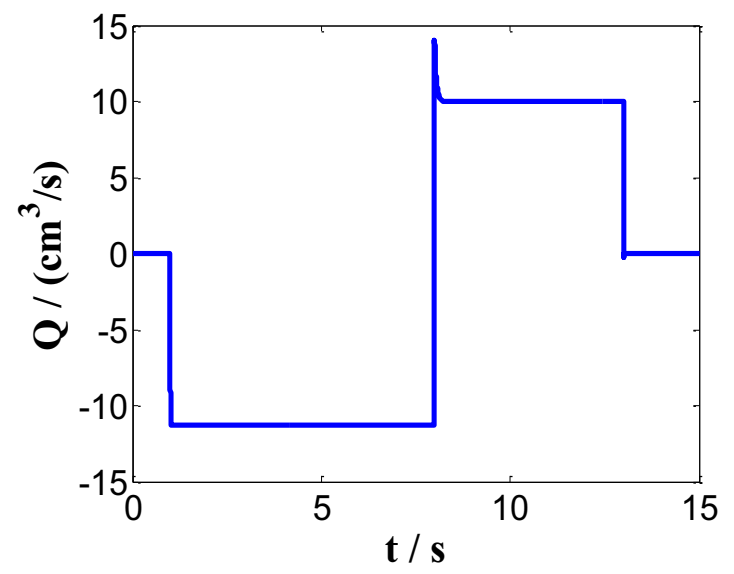

Figure 10. Flow of 4-way valve port2

Above the flow analysis of the relief valve and the 4-way valve is used to solve the problem, that it is difficult to be measured for the flow at the mechanism working.
For it is difficult to place the sensors in the actual machine, the parameters of the flow is not easy achieved. It is feasible and effective to calculate the flow parameters through the simulation analysis. It provides the basis and reference for the further research of hydraulic system and related properties.

\section{Conclusion}

The virtual prototyping model of elevating equilibrator is established by Solidworks and imported into ADAMS. The hydraulic control system model is built in EASY5, and co-simulation is executed through dynamics data exchange. The models are in agreement with actual working situation and can provide reference for related research in the future. The direction of oil flow changing, the shock force to piston rod is of significant influence on the working performance and service life and should be considered on the design and calibration.

Using this model, the flow parameters of the relief valve and the valve are solved. It is difficult to add sensors in a complex mechanism when the actual mechanical is working. So the virtual prototyping simulation technology can be used to measure different parameters. This provides a new solution to solve practical engineering problems and can provide reference and basis for fault diagnosis and so on.

\section{References}

1. Sheng Longbo, MA Jisheng. Co-simulation of the Braking System of a Tracked Vehicle Based on ADAMS and EASY5[J]. Journal of Ordnance Engineering College (2012).

2. CHENG Xuejing, WANG Qing'an. Co-simulation of $40 \mathrm{k} \mathrm{N}$ Hydraulic Crane Based on ADAMS and EASY5 [J]. Machine Tool \& Hydraulics (2015).

3. Lv Yingbo. Solidworks 2016 Technical Manual[M](2016).

4. Li Wenji. Application of Aviation Hydraulic Oil[M].68 (1995).

5. Zhou Jie, MA Jisheng. Dynamic Simulation Research on Mechanical-Hydraulic Coupling of a Certain Weapon Launch Tower's Pendulum System[J]. Machine Tool \& Hydraulics (2014).

6. Zheng Jianrong. ADAMS: Introduction and Improvement of Virtual Prototyping Technology[M] (2002). 